\title{
Analysis of the Transmission Performance Limits for a Multi-layer Transmitarray Unit Cell
}

\author{
Zhishu Qu, James R. Kelly, Member, IEEE, Yue Gao, Senior Member, IEEE
}

\begin{abstract}
This communication presents a theoretical study that establishes the performance limits for a multi-layer transmitarray unit cell. This is the first study to be applicable to unit cells in which the conducting resonators, on the different layers, are shaped differently. A theoretical calculation is derived at the beginning. The theoretical calculations predict that, for an $S_{21}$ amplitude of $-1 \mathrm{~dB}$, unit cells having two and three conducting layers provide a phase shifting range of $170^{\circ}$ and $360^{\circ}$, respectively. Additionally, for a given phase shifting range of $S_{21}$, a new methodology for analyzing the maximum $S_{21}$ amplitude, based on different substrates, is proposed. For the first time, we prove that it is efficient to attain the maximum $S_{21}$ amplitudes by employing a smaller substrate permittivity or a quarter-of-wavelength substrate electrical thickness. Finally, the theoretical calculations have been validated through computer simulation.
\end{abstract}

Index Terms-Multi-layer transmission unit cell, phase shifting range, transmitarray antenna, transmission performance.

\section{INTRODUCTION}

High-gain beam steerable antennas are required for use in a range of emerging applications, including: satellite internet, and 5G mobile access at mmWave frequencies. Those antennas must offer high efficiency together with a wide scan angle range. Phased array antennas are amongst the most popular form of high-gain beam steerable antenna. A disadvantage of phased array antennas is that they require a complex feed network which suffers from severe energy losses. This impedes their use at high frequencies (i.e. mmWave or $\mathrm{THz}$ ) or in large aperture antennas. By contrast, the aperture of a transmitarray antenna is spatially illuminated by a single element feed. This type of feed is simpler and more energy efficient than that of a phased array [1]-[7].

Generally, for a transmitarray antenna, if it is expected to steer the beam to wide angles and also to maintain high gain, then this imposes three important requirements on the unit cell of the transmitarray antenna: 1) it must be possible to vary the phase of $S_{21}$ (i.e. $\angle \mathrm{S}_{21}$ ) over a wide range; 2 ) the amplitude of $\mathrm{S}_{21}$ must remain high throughout the tuning range, otherwise the efficiency and hence gain of the antenna will be adversely effected; 3) there should be only a small fluctuation in the amplitude of $S_{21}$ over the entire phase shifting range of the unit cell. From this discussion, it is clear that the phase shifting range that can be achieved within a unit cell of the transmitarray antenna along with the corresponding amplitude of $S_{21}$ both play a vitally important role in determining the beam steering performance and efficiency of a transmitarray antenna. This motivates the study reported in this communication. Henceforth, we

Manuscript is submitted on 27, April, 2021. This work was supported in part by a grant from The United Kingdom Engineering and Physical Sciences Research Council, EPSRC, under grant number: EP/P008402/1 and EP/P008402/2, and in part by the Chinese Scholarship Council who are funding the first author's PhD studentship.

Zhishu Qu and James R. Kelly are with The Antennas \& Electromagnetics Research Group, which is based in The School of Electronic Engineering and Computer Science, Queen Mary University of London, Mile End Road, London (email: z.qu@qmul.ac.uk; j.kelly@qmul.ac.uk).

Yue Gao is with The Wireless Communications Group, which is based in The Institute for Communication Systems, University of Surrey, U.K. refer to the unit cells of the transmitarray antenna.

For a unit cell within a transmitarray antenna, to compensate for phase changes caused by the spatial separation, it is expected to provide a $360^{\circ}$ phase shifting range. However, it is difficult to achieve this goal using a unit cell comprising only one or two conducting layers [3]. For this reason, larger numbers of conducting layers, separated by dielectrics or air gaps, are commonly employed [8]-[11]. For example, seven and five conducting layers are employed in [8] and [9], respectively, to cover a $360^{\circ}$ phase shifting range. To provide the required phase shifting range and yet maintain an $S_{21}$ amplitude $\leq-1 \mathrm{~dB}$, the unit cells in [8]-[11] require at least four identical conducting layers. Although increasing the number of layers within the unit cell will normally expand the phase shifting range, one potential drawback of such an approach is the relatively high profile of the unit cell. Furthermore, increasing the number of layers will inevitably decrease the amplitude of $S_{21}$. In an effort to reduce the profile, unit cell designs having only three conducting layers while still providing the required phase shifting range are proposed in [12] and [13]. However, [12] and [13] focus on the geometries of the unit cell designs, and do not discuss the limitations on the phase shifting range.

Most of the published literature, on unit cells for transmitarray antennas, presents designs having a specific geometry that was optimized to provide either: 1) a large phase shifting range, 2) the maximum amplitude of $S_{21}$, or 3) a low profile. However, these parameters are interrelated. For this reason, optimizing one parameter will often have an adverse effect on the other parameters. For example, [8] presents a unit cell having a $360^{\circ}$ phase shifting range. The unit cell comprises seven conducting layers and achieves a large phase shifting range at the expense of a higher profile. More importantly, the transmission behavior of the transmitarray antenna unit cells is usually neglected in the literature. [3] presents an investigation into the phase shifting range of a multi-layer transmitarray antenna unit cell. However, the conclusion drawn in [3] is only valid for unit cells in which the resonators on each of the conducting layers have identical geometries. The challenge of investigating the transmission behavior of a transmitarray unit cell for more general cases is not solved. This communication presents a more general study which, for the first time, applies to unit cells in which the resonators on the various conducting layers have different geometries, size, orientation, or all three.

\section{Theoretical Calculation of the Phase Shifting Range for TransmitarRay Unit Cells}

This section presents the derivation of equations that can be used to determine a theoretical limit on the phase (of $S_{21}$ ) shifting range for the unit cells within a multi-layer transmitarray antenna. Based on this theoretical analysis, we have calculated the phase shifting range for unit cells having two and three conducting layers. The phase shifting range depends on the $S_{21}$ amplitude that can be tolerated. We present the phase shifting range pertaining to an $S_{21}$ amplitude of $-1 \mathrm{~dB}$. 


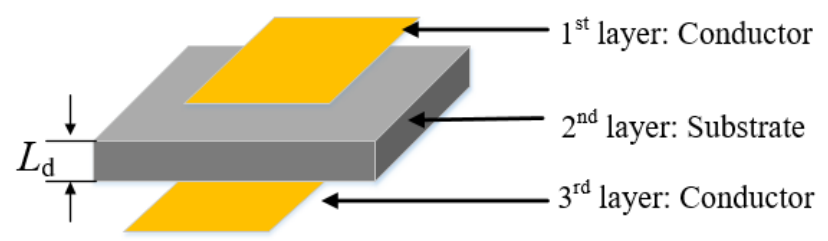

Fig. 1. Schematic illustration of a unit cell incorporating two conducting layers.

The proposed calculation process is valid for unit cells incorporating resonators which differ in their: size, geometry, orientation, or all three.

\section{A. Theoretical Calculation for A Unit Cell Having Two Conducting Layers}

A single conducting layer can be regarded as a two-port network [14], [15]. According to linear two-port network theory [16], the scattering matrix ([S] matrix), for the single conducting layer, can be simplified as following (1) and (2). The detailed derivation process was written in [3].

$$
\begin{aligned}
& S_{11}=S_{22}=\sin \left(\angle S_{21}\right) \mathrm{e}^{j\left(\angle S_{21} \pm \frac{\pi}{2}\right)} \\
& S_{12}=S_{21}=\cos \left(\angle S_{21}\right) \mathrm{e}^{j\left(\angle S_{21}\right)}
\end{aligned}
$$

From (1) and (2), we can see that the $[\mathrm{S}]$ matrix is a function of only one variable, namely phase of $S_{21}\left(\angle S_{21}\right)$.

Fig. 1 shows a unit cell for a transmitarray antenna incorporating two conducting layers. The two conducting layers are printed on the opposite sides of a single dielectric substrate. Each of the conducting layers can be modelled by a separate [S] matrix. If the resonators on the two conducting layers are identical in size, geometry and orientation, then their [S] matrices will also be identical. If they differ in any of these three respects, then so will their [S] matrices. This communication investigates a general case in which the [S] matrices on each layer differ.

Note that each conducting layer is represented by a separate [S] matrix. Each of the dielectric substrates is also represented by a separate $[\mathrm{S}]$ matrix. The matrices for each conducting layer are given by (1) and (2). Whereas the matrix for the dielectric substrate is given by (3) and (4).

$$
\begin{gathered}
S_{11}=S_{22}=\frac{\Gamma\left(1-\mathrm{e}^{-j 2 \beta L_{d}}\right)}{1-\Gamma^{2} \mathrm{e}^{-j 2 \beta L_{d}}} \\
S_{12}=S_{21}=\frac{\left(1-\Gamma^{2}\right) \mathrm{e}^{-j \beta L_{d}}}{1-\Gamma^{2} \mathrm{e}^{-j 2 \beta L_{d}}} \\
\Gamma=\frac{1-\sqrt{\varepsilon_{r}}}{1+\sqrt{\varepsilon_{r}}} \text { and } \beta=\frac{2 \pi \sqrt{\varepsilon_{r}}}{\lambda_{0}}
\end{gathered}
$$

Where: $\varepsilon_{r}$ is the permittivity of the substrate, $L_{d}$ is the physical thickness of the substrate, and $\lambda_{0}$ is the free-space wavelength. From (3), (4) and (5), it can be seen that the [S] matrix of a particular substrate is determined by the permittivity and thickness of the substrate material, as one would expect. However, these two factors have a similar influence on the $[\mathrm{S}]$ matrix. For brevity, we will only explore the effect of varying the substrate permittivity whilst keeping the substrate thickness constant. The overall [S] matrix, for a multilayer unit cell, can be obtained by cascading the [S] matrices for the individual layers. (6) to (8) result when two separate [S] matrices are cascaded together.

$$
S_{11}^{c}=\frac{S_{11}^{2} S_{12}^{1} S_{21}^{1}}{1-S_{11}^{2} S_{22}^{1}}+S_{11}^{1}
$$

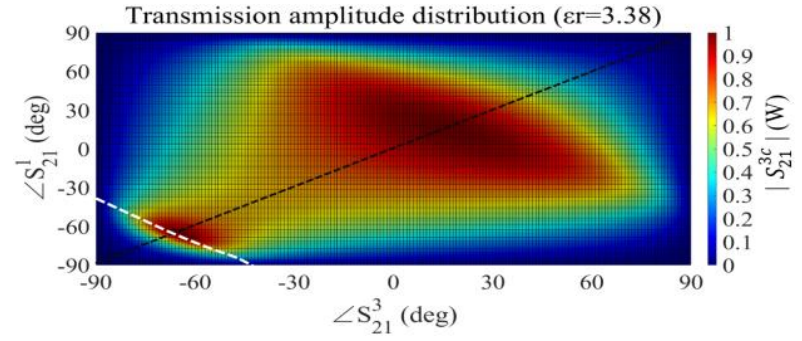

(a)

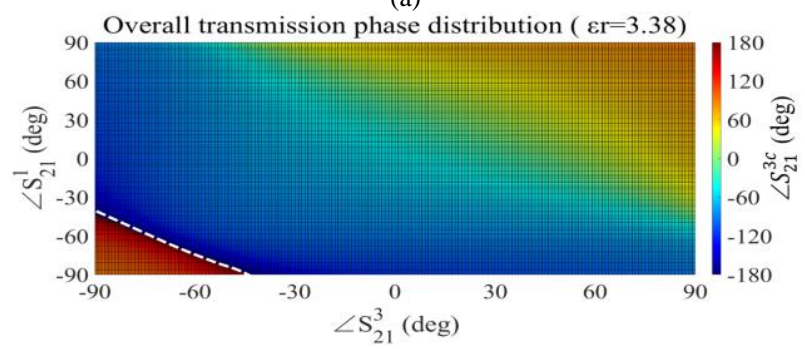

(b)

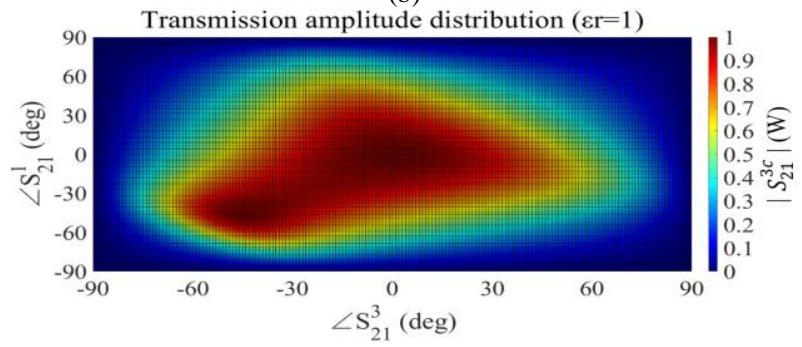

(c)

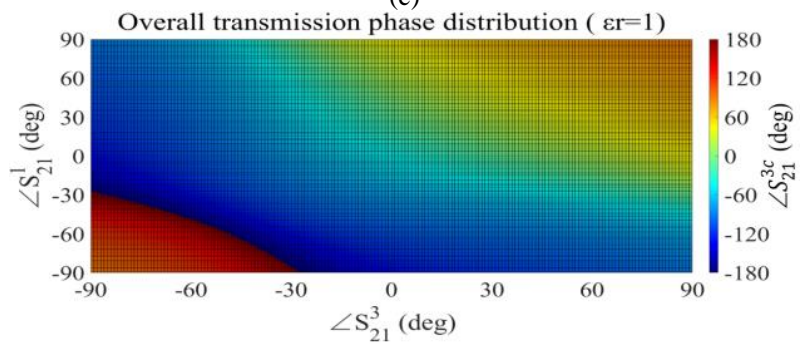

(d)

Fig. 2. Amplitude and phase distributions as a function of two variables $\left(\angle \mathrm{S}^{1}{ }_{21}, \angle \mathrm{S}^{3}{ }_{21}\right)$. (a) and (c) represent the amplitude distributions with substrate permittivities of 3.38 and 1 , respectively. (b) and (d) represent the phase distributions with substrate permittivities of 3.38 and 1 , respectively. The electrical thickness $\left(\beta L_{d}\right)$ remains fixed at $45^{\circ}$.

$$
\begin{aligned}
& S_{12}^{c}=S_{21}^{c}=\frac{S_{21}^{1} S_{21}^{2}}{1-S_{11}^{2} S_{22}^{1}} \\
& S_{22}^{c}=\frac{S_{22}^{1} S_{21}^{2} S_{12}^{2}}{1-S_{11}^{2} S_{22}^{1}}+S_{22}^{2}
\end{aligned}
$$

Where: $S^{l}, S^{2}, S^{c}$ represent the first matrix, the second matrix and the overall cascaded matrix, respectively.

For a transmitarray antenna unit cell comprising two conducting layers, the overall $[\mathrm{S}]$ matrix can be determined by repeating the cascading process twice. Based on (7), the overall $S_{21}$ is given as follows:

$$
S_{21}^{3 c}=\frac{S_{21}^{2 c} \cdot S_{21}^{3}}{1-S_{11}^{3} \cdot S_{22}^{2 c}}
$$

Where $S^{2 c}, S^{3 c}$ represent the [S] matrices obtained by cascading two and three separate [S] matrices, respectively. $S^{3}$ represents the [S] matrix of the third layer of the unit cell. Note that, $S_{21}^{2 c}, S_{22}^{2 c}$ are given by (7) and (8), respectively. 


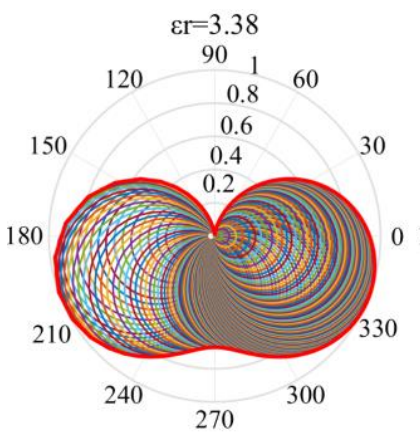

(a)

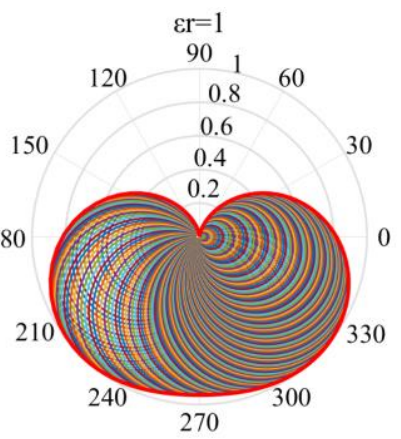

(b)
Fig. 3. Relationship of the $S_{21}$ amplitude and phase $\left(\beta L_{d}=45^{\circ}\right)$. (a) shows the relationship with the permittivity of 3.38. (b) shows the relationship with the permittivity of 1

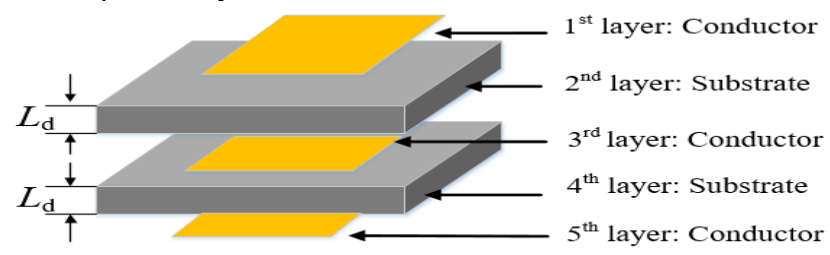

Fig. 4. Schematic structure of a unit cell having three conducting layers.

(10) is obtained by substituting (7), and (8) into (9).

$$
\begin{aligned}
S_{21}^{3 c} & =\frac{S_{21}^{1} \cdot S_{21}^{2} \cdot S_{21}^{3}}{\left(1-S_{11}^{2} \cdot S_{22}^{1}\right)\left[1-S_{11}^{3} \cdot\left(\frac{S_{22}^{1} \cdot S_{21}^{2} \cdot S_{12}^{2}}{1-S_{11}^{2} \cdot S_{22}^{1}}+S_{22}^{2}\right)\right]} \\
& =\frac{S_{21}^{1} \cdot S_{21}^{2} \cdot S_{21}^{3}}{1-S_{11}^{1} \cdot S_{11}^{2}-S_{11}^{2} \cdot S_{11}^{3}-S_{11}^{1} \cdot S_{11}^{3} \cdot\left[\left(S_{21}^{2}\right)^{2}-\left(S_{11}^{2}\right)^{2}\right]}
\end{aligned}
$$

In practice, once a substrate has been selected, its properties (and hence [S] matrix) are fixed. Consequently, for a given choice of substrate, the main factors in (10) that we can control are the [S] matrices of the two conducting layers. The $[\mathrm{S}]$ matrix of each conducting layer is a function of only one variable (i.e. $\angle S_{21}$ ). However, the overall relationship, described by (10), is actually a function of two variables (i.e. $\angle S_{21}^{1}, \angle S_{21}^{3}$ ). (10) can, therefore, simply be expressed as follows:

$$
\text { Am } \cdot e^{\text {jPhase }}=S_{21}^{3 c}=f\left(\angle \mathrm{S}_{21}^{1}, \angle S_{21}^{3}\right)
$$

For each set of $\angle S_{21}^{1}$ and $\angle S_{21}^{3}$ values, there is a corresponding complex value of $S_{21}^{3 c}$. Fig. 2 plots the $S_{21}^{3 c}$ amplitude and phase distributions as a function of these two variables $\left(\angle S_{21}^{1}, \angle S_{21}^{3}\right)$. The different parts of Fig. 2 pertain to different substrate permittivity values. The electrical thickness $\left(\beta L_{d}\right)$ remains fixed. Figs. 2(a) and (b) show the overall $S_{21}$ amplitude and phase distributions, respectively, when the permittivity is set to 3.38. Similarly, Figs. 2(c) and (d) show the separate $S_{21}$ amplitude and phase distributions when the permittivity is set to 1 . The phase of the overall $S_{21}$ (i.e. $\angle S_{21}^{3 c}$ ) remains constant at all points on the white dashed line, shown in Fig. 2(b). The corresponding amplitude of the overall $S_{21}$ (i.e. $\left|S_{21}^{3 c}\right|$ ) is indicated by the points on the white dashed line, shown in Fig. 2(a). It can be seen that amplitude assumes several different values. From the $S_{21}$ amplitude distributions, shown in Figs. 2(a) and (c), it can be seen that the maximum amplitude for a given $\angle \mathrm{S}_{21}$ value is obtained when the values of the two variables are identical (as indicated by the diagonal dashed line in Fig. 2(a)). In other words, for a given $\angle \mathrm{S}_{21}$, the maximum amplitude of the overall $\mathrm{S}_{21}$ is obtained when the separate $[\mathrm{S}]$ matrices associated with the different layers are identical to one another.

Fig. 3 plots the relationship between the $S_{21}$ amplitude and phase in polar coordinates. The relationship shown in Fig. 3 is for the

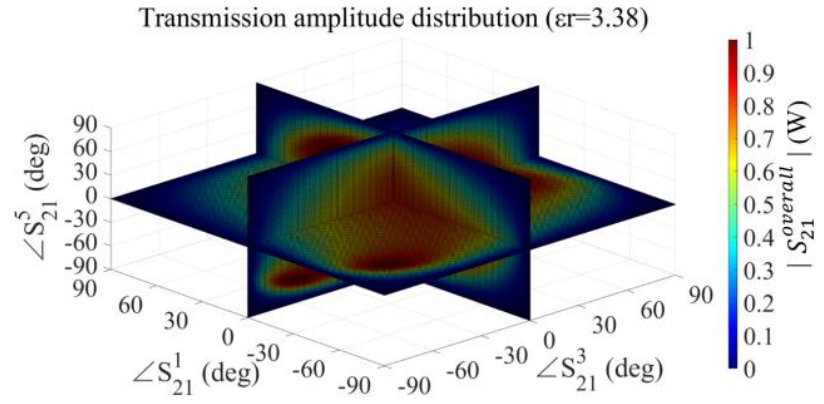

(a)

Transmission phase distribution $(\varepsilon r=3.38)$

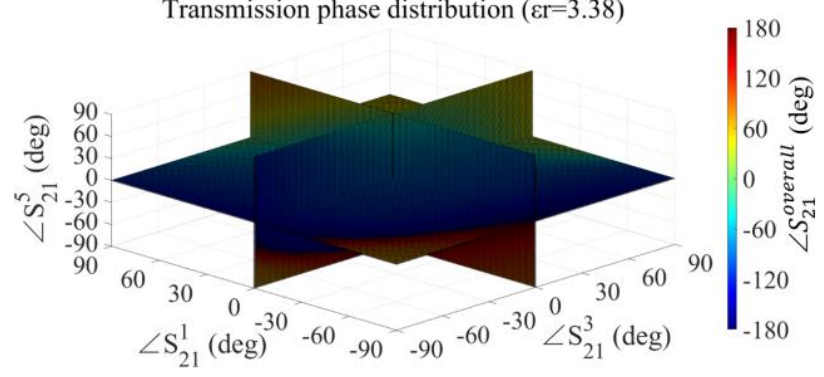

(b)

Transmission amplitude distribution $(\varepsilon \mathrm{r}=1)$

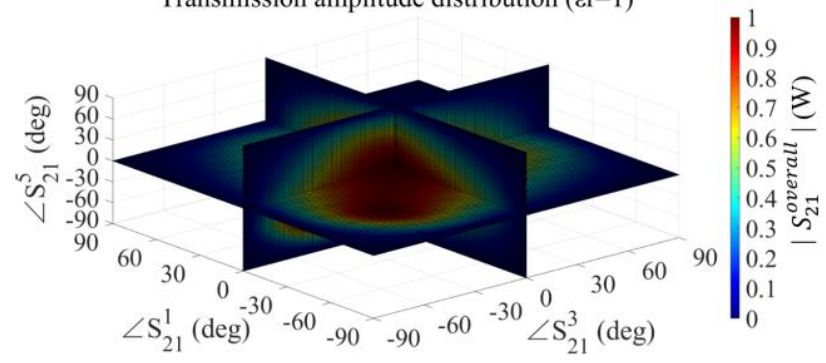

(c)

Transmission phase distribution $(\varepsilon \mathrm{r}=1)$

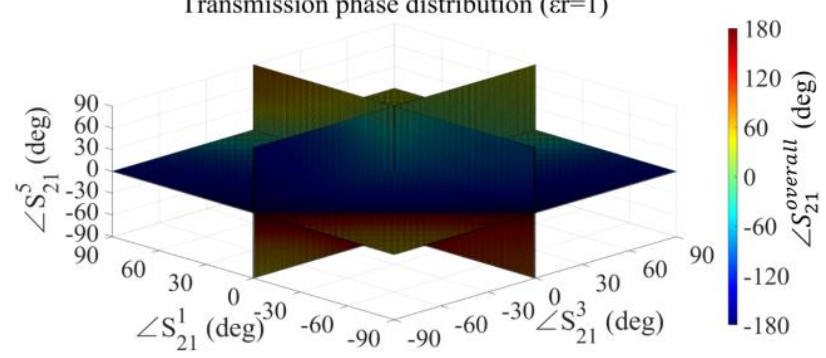

(d)

Fig. 5. 3D views of the overall amplitude and phase distributions of $S_{21}$ as a function of three variables. The different parts of the figure relate to different permittivity values but constant electrical thicknesses $\left(\beta L_{d}\right)=$ $45^{\circ}$. (a) and (c) show the amplitude distributions, and (b) and (d) show the phase distributions.

general case in which the resonators on each conducting layer have different: sizes, geometries, orientations or perhaps all three. In Fig. 3 , the curves having a bold red outline represent the case that the resonators on the two conducting layers are identical in every way. From Fig. 3, we can draw an interesting and valuable conclusion, namely that the $S_{21}$ amplitude for the overall structure attains its optimal value when the geometry of the resonators on the two conducting layers are identical in every way. This conclusion agrees well with the conclusion obtained from Fig. 2. According to [3], for a unit cell incorporating two identical conducting layers, the limit of the phase shifting range for a $-1 \mathrm{~dB}$ amplitude of $\mathrm{S}_{21}$ is $170^{\circ}$. We generalize the analysis to conducting layers incorporating resonators that have different: sizes, geometries, orientations or perhaps all 


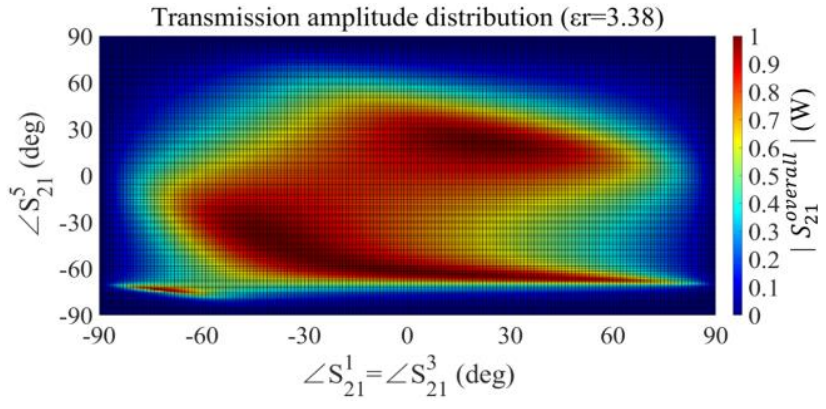

(a)

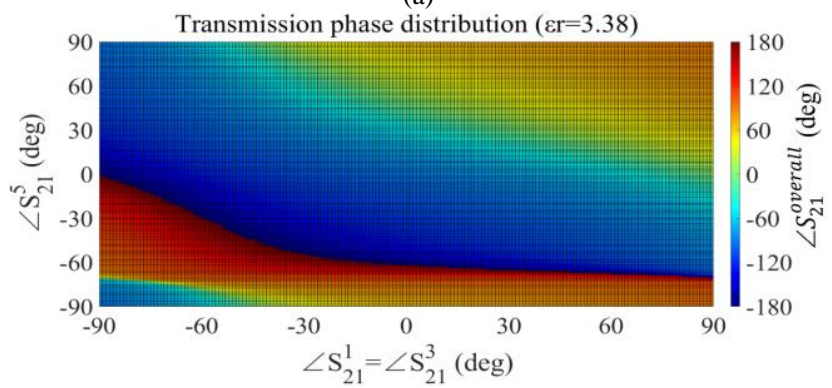

(b)

Fig. 6. Amplitude and phase distributions on $2 \mathrm{D}$ cut-plane $\left(\beta L_{d}=45^{\circ}\right)$.

three. We discover that having the ability to vary those things does not increase the phase shifting range of a unit cell having two conducting layers.

\section{B. Theoretical Calculation for A Unit Cell Having Three Conducting Layers}

Based on the analysis presented in Section II-A, it is clear that a unit cell having two conducting layers cannot achieve a phase shifting range of $360^{\circ}$ for a $-1 \mathrm{~dB}$ amplitude. For that reason, we are forced to consider a unit cell having three conducting layers. In such a unit cell, the three conducting layers are separated by two substrates, as shown in Fig. 4. To simplify the calculations, we assume that the permittivity and electrical thickness of the two dielectric substrates are identical and fixed. This implies that the [S] matrices of the two dielectric substrates are identical and remain constant. Thus, we use three variables, i.e. $\angle S_{21}^{1}, \angle S_{21}^{3}, \angle S_{21}^{5}$, to represent the $[\mathrm{S}]$ matrices of three conducting layers, respectively.

The overall $[\mathrm{S}]$ matrix, for the unit cell having three conducting layers, is formed by cascading the $[\mathrm{S}]$ matrix of a unit cell having two conducting layers with that of the additional conducting layer and substrate. The overall $S_{21}$ of the unit cell having three conducting layers can be expressed as a function of three variables using (12). Note that the form of (12) is similar to that of (11).

$$
\text { Am } \cdot e^{\text {jPhase }}=S_{21}^{\text {overall }}=f\left(\angle \mathrm{S}_{21}^{1}, \angle S_{21}^{3}, \angle S_{21}^{5}\right)
$$

Fig. 5 shows $3 \mathrm{D}$ plots illustrating the amplitude and phase distribution of $S_{21}$ as functions of three variables. Each plot shows three cuts through the 3D distribution. The different plots, shown in Fig. 5, pertain to different dielectric substrate permittivity values but constant electrical thicknesses. Figs. 5(a) and (c) show the overall $S_{21}$ amplitude distributions pertaining to dielectric substrate permittivity values of 3.38 and 1, respectively. Figs. 5(b) and (d) show the corresponding phase distributions of the overall structure. The phase (of $S_{21}$ ) shifting range covers $360^{\circ}$ with an $S_{21}$ amplitude of $-1 \mathrm{~dB}$. This is evident from the 2D cut-plane images in Fig. 6. Fig. 6 shows the amplitude and phase distributions for the case where the first and third conducting layers have identical [S] matrices (i.e. $\left.\angle S_{21}^{1}=\angle S_{21}^{3}\right)$. The permittivity is 3.38 and the electrical thickness
For the fixed-substrate structure

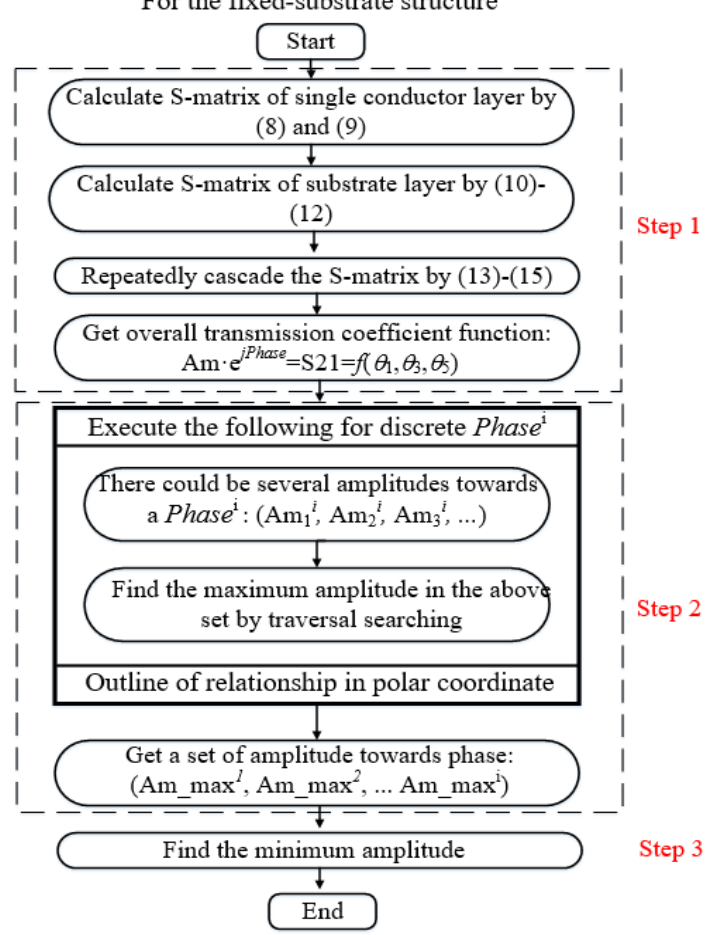

Fig. 7. Flowchart of the calculation process.

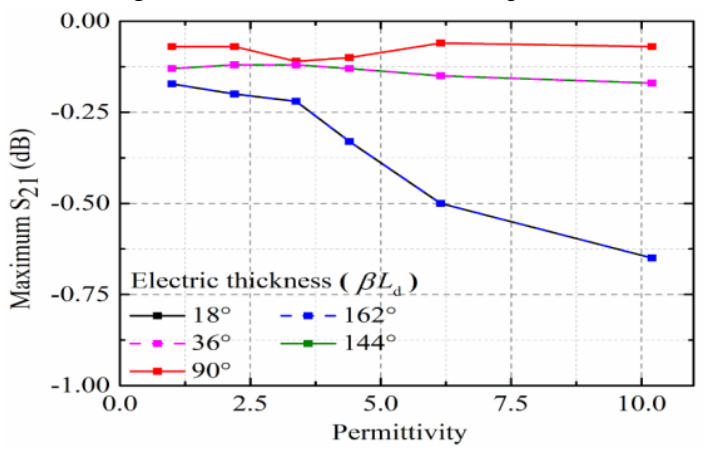

Fig. 8. The maximum $S_{21}$ amplitude values as a function of the permittivity of the dielectric substrate.

$\left(\beta L_{d}\right)$ is $45^{\circ}$. We have also conducted further investigations for different substrate permittivity values, and found that the phase shifting range, for most of the permittivity values considered (studied range: 1 to 10 ), can cover $360^{\circ}$.

In the previous literature, for the unit cells incorporating identical resonators on different conducting layers, at least four conducting layers were required to provide a $360^{\circ}$ phase shifting range with an $\mathrm{S}_{21}$ amplitude of $-1 \mathrm{~dB}$. However, in this communication, we prove that the phase shifting range can be improved by employing resonators, on the different layers, which differ in their: size, geometry, orientation, or all three. Specifically, we discovered that a unit cell having three independently conducting layers can provide a $360^{\circ}$ phase shifting range with an $S_{21}$ amplitude of $-1 \mathrm{~dB}$.

\section{Theoretical CALCUlation of THE MAXimum Transmission AMPLITUDE FOR TRANSMITARRAY UNIT CELLS}

In this section, we fix the phase shifting range of the unit cells and evaluate the amplitude of $S_{21}$ for a multi-layer unit cell as a function of the substrate parameters. We focus on a unit cell, for a transmitarray antenna, incorporating three conducting layers. In Section II-B, we showed that such a configuration can yield a phase 


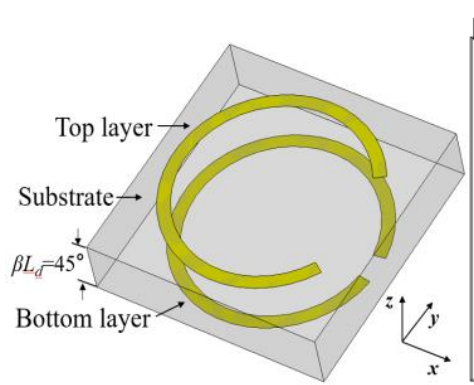

(a)

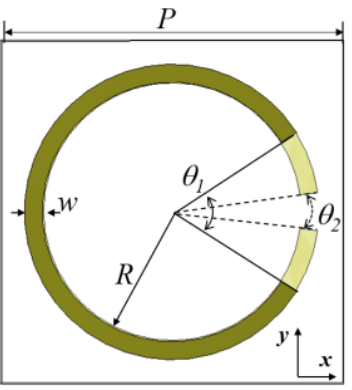

(b)
Fig. 9. Geometry and dimensions of Design \#1. (a) Perspective view; (b) Top view. The uppermost layer of metal is shown in dark yellow and the lowermost layer of metal is shown in light yellow.

shifting range of $360^{\circ}$. We propose a new methodology for calculating the maximum $S_{21}$ amplitude. Based on this newly proposed method, we calculate the $S_{21}$ amplitude for different substrates.

Based on the analysis presented in Section II-B, it is known that for a unit cell having three conducting layers and providing a $360^{\circ}$ phase shifting range, the maximum $S_{21}$ amplitude is determined by the permittivity and electrical thickness of the dielectric substrate. Consequently, for a unit cell based around a given dielectric substrate, the process of calculating the maximum $S_{21}$ amplitude comprises three steps. The first step is to calculate the $S_{21}$ of the overall cascaded structure, and obtain a function of three variables similar to (12), shown in Section II-B. Secondly, we must find the maximum $S_{21}$ amplitude pertaining to each phase shift value. Note that a given phase of $S_{21}\left(\angle S_{21}\right)$ usually corresponds to several different amplitude values as can be seen in Fig. 2, shown in Section II-A. For this reason, the second step was achieved by applying a tree traversal algorithm, i.e. by searching and comparing all of the $\mathrm{S}_{21}$ amplitude values pertaining to a fixed value of $\angle \mathrm{S}_{21}$. By repeating this process for a set of phase shift values, we obtained a corresponding set of amplitude values. Finally, the third step is to find the minimum value from the set of values obtained in step 2 . The result is actually the maximum $S_{21}$ amplitude over the considered phase shifting range. If the dielectric substrates were changed, then the calculation process, discussed above, would need to be repeated. Fig. 7 gives a flowchart summarizing the whole calculation process. Fig. 8 presents the maximum $\mathrm{S}_{21}$ amplitude values associated with a $360^{\circ}$ phase shifting range for different dielectric substrates. Consider Fig. 8 and assume that the substrate permittivity is fixed. Setting the total electrical thickness of the unit cell equal to a quarter of a wavelength usually yields the maximum amplitude value. Meanwhile, for the unit cells based on very thin or very thick substrates, the $S_{21}$ amplitude drops sharply as the permittivity increases.

\section{SiMULATION RESULTS}

This section presents the results of a study performed within the computer simulation environment. The purpose of the study was to validate the phase shifting range of the multi-layer unit cells, presented in Section II. These unit cells were simulated at $2.5 \mathrm{GHz}$.

All of the computer simulation results, reported in this communication, were obtained using CST Microwave Studio 2019.

\section{A. Demonstration of A Unit Cell Having Two Conducting Layers}

Fig. 9 shows the structure of a unit cell that was designed for the purpose of validating the results presented in Section II-A. For

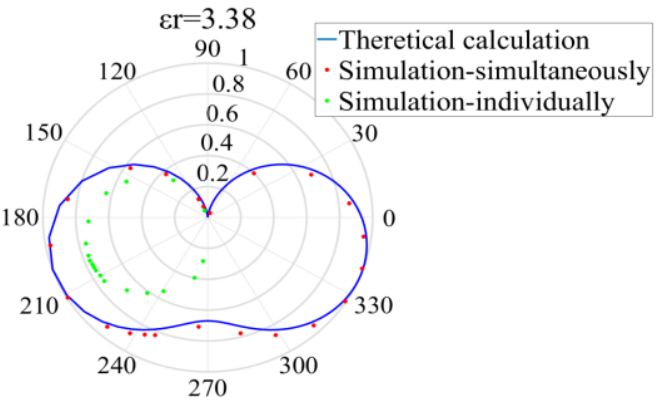

(a)
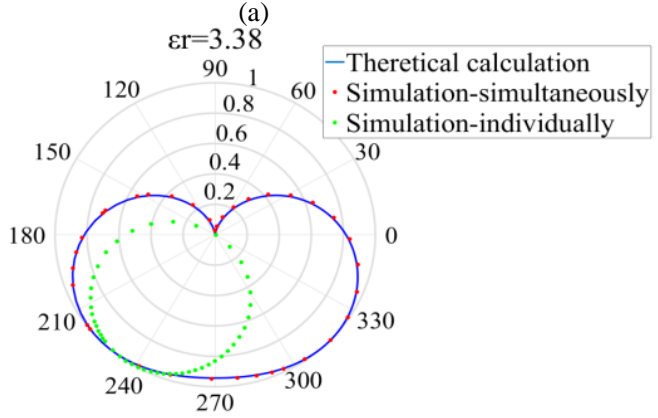

(b)

Fig. 10. The relationship between the $S_{21}$ amplitude and phase for Design $\# 1$. Permittivity is a parameter. $\left(\beta L_{d}=45^{\circ}\right)$

convenience, we will refer to this as Design \#1. Design \#1 incorporates two conducting layers that are located on the opposite sides of a dielectric substrate. The resonators on each of the conducting layers are shaped in the form of a split-ring. The parameters of the resonator are as follows: $\mathrm{R}=14.5 \mathrm{~mm}, \mathrm{w}=2 \mathrm{~mm}$. The resonators operate at a central frequency of $2.5 \mathrm{GHz}$, and the periodicity of the unit cell is $0.32 \lambda_{0}$, where: $\lambda_{0}$ is the free-space wavelength $(\mathrm{P}=38.4 \mathrm{~mm})$. The substrate has an electrical thickness $\left(\beta L_{d}\right)$ of $45^{\circ}$. The electrical thickness of the substrate was not varied during the study. $\theta_{1}$ and $\theta_{2}$ represent the angles within the split ring resonators on the uppermost and lowermost conducting layers, respectively. By separately tuning the angles of these gaps within the allowed range (i.e. $\theta_{1}: 5^{\circ}$ to $235^{\circ} ; \theta_{2}: 5^{\circ}$ to $235^{\circ}$ ), the [S] matrix of each conducting layer may be varied independently. We compare the results obtained through calculation against those obtained through computer simulation in CST Microwave Studio. Fig. 10 shows the relationship between the overall amplitude and phase of $S_{21}\left(\angle S_{21}\right)$ for different permittivity values. The red dotted curves represent the behavior of unit cells in which the geometries of the resonators on both conducting layers are varied in sympathy with one another. The green curves depict the behavior of unit cells in which the geometries of the resonators on both conducting layers are varied independently. From Fig. 10, it can be seen that the simulation results agree well with the theoretical calculations. The best amplitude performance, for a unit cell incorporating two conducting layers, corresponds to the situation in which the resonators, on the different layers, are identical in: geometry, size, and orientation, as expected. Agreement between simulation and theoretical results is better when the substrate permittivity is low as shown in Fig. 10(b). At some points in Fig. 10(a), there is a small deviation between the simulation results and those predicted by theory. This deviation can be attributed to the higher-order mode coupling between layers which is accounted for within the simulations but not modelled by the theoretical analysis.

B. Demonstration of A Unit Cell Having Three Conducting Layers 


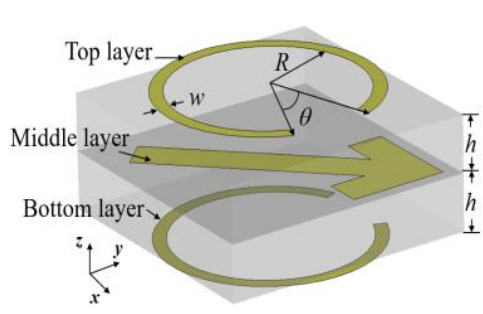

(a)

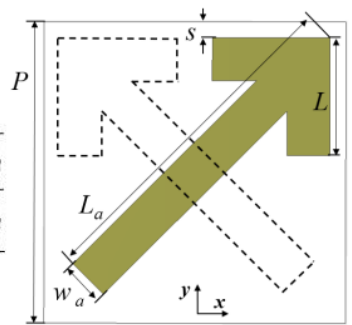

(b)
Fig. 11. Geometry and dimensions of Design \#2. (a) Perspective view. (b) Top view of the middle layer and its mirrored image shown by the dashed outline. $\mathrm{P}=38.4, \mathrm{R}=13.5, \mathrm{w}=2, \theta=15^{\circ}, \mathrm{h}=7.6, \mathrm{~s}=2, \mathrm{w}_{\mathrm{a}}=5.5, \mathrm{~L}_{\mathrm{a}}=$ 38.4. All dimensions are in $\mathrm{mm}$. The dielectric substrates have a relative dielectric constant of 3.38 .

Fig. 11 shows the structure of a unit cell that was designed for the purpose of validating the results presented in Section II-B. For convenience, we will refer to this as Design \#2. Design \#2 incorporates three conducting layers and 2 dielectric substrates. Design \#2 incorporates different resonator geometries on each of the three conducting layers. The split ring resonators, on the uppermost and lowermost conducting layers, have the same size but different orientations. An arrow-shaped conductor, as illustrated by the solid line in Fig. 11(b), is orientated diagonally and located on the middle layer. The length of the arrow head $(L)$ is varied from $10 \mathrm{~mm}$ to 29 $\mathrm{mm}$ in order to vary the phase shift through the structure. Fig. 12 presents the simulation results for the $\mathrm{S}_{21}$ amplitude and phase versus frequency. From Fig. 12, we can see that for an $S_{21}$ amplitude of -1 $\mathrm{dB}$, a $\sim 230^{\circ}$ phase shifting range is achieved at $2.5 \mathrm{GHz}$. An additional $180^{\circ}$ phase shift is obtained by simply mirroring the arrow-shaped resonator about the Y-axis. The resulting structure is shown by the dash outline, in Fig. 11(b). The total phase shift for the resulting unit cell is then over $360^{\circ}$. For that reason, the proposed unit cell validates the theoretical calculations. Note that for a phase shifting range of $360^{\circ}$, the maximum $S_{21}$ amplitude of the proposed unit cell is actually $-0.7 \mathrm{~dB}$ ( $L: 18 \mathrm{~mm}$ to $28 \mathrm{~mm})$ which, as expected, is worse than the theoretical amplitude limit calculated in Section III.

\section{CONCLUSION}

This communication presents theoretical calculations which yield the transmission performance limits for multi-layer transmitarray antenna unit cells. To the best of our knowledge, this paper is the first to analyze the transmission behavior of unit cells incorporating resonators that have different: sizes, geometries, orientations or perhaps all three on the different layers. Based on the proposed analysis, for an $S_{21}$ amplitude of $-1 \mathrm{~dB}$, the phase shifting ranges of a unit cell having two conducting layers and three conducting layers are $170^{\circ}$ and $360^{\circ}$, respectively. These phase shift values are achieved when the geometries of the resonators on the different layers are varied in sympathy and independently, respectively. Additionally, we also propose a new method for calculating the maximum $\mathrm{S}_{21}$ amplitude for a $360^{\circ}$ phase shifting range. The calculation was performed as a function of the thickness and permittivity of the dielectric substrate. For the first time, the paper explains why a dielectric substrate having a higher permittivity usually leads to a lower $S_{21}$ amplitude. The study shows that the optimum electrical thickness, for a unit cell, is a quarter-of-awavelength. These conclusions are validated through computer simulation. The proposed methodologies are valuable to guide future transmitarray unit cell designs.

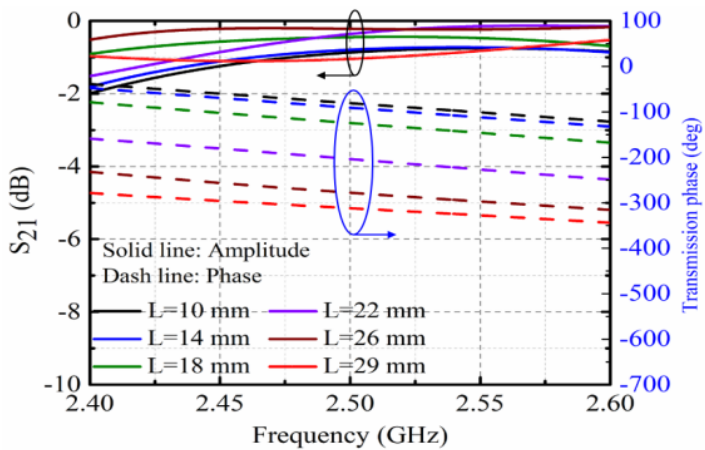

Fig. 12. $S_{21}$ amplitude and phase versus frequency of some representative parameters.

[1] G. Liu, H.-J. Wang, J.-S. Jiang, F. Xue, and M. Yi, “A high-efficiency transmitarray antenna using double split ring slot elements," IEEE Antennas Wireless Propag. Lett., vol. 14, pp. 1415-1418, 2015.

[2] B. Rahmati and H. R. Hassani, "High-efficient wideband slot transmitarray antenna," IEEE Trans. Antennas Propag., vol. 63, no. 11, pp. 5149-5155, Nov. 2015.

[3] A. H. Abdelrahman, A. Z. Elsherbeni, and F. Yang, "Transmission phase limit of multilayer frequency-selective surfaces for transmitarray designs," IEEE Trans. Antennas Propag., vol. 62, no. 2, pp. 690-697, Feb. 2014.

[4] A. H. Abdelrahman, A. Z. Elsherbeni, and F. Yang, "Transmitarray antenna design using cross-slot elements with no dielectric substrate," IEEE Antennas Wireless Propag. Lett., vol. 13, pp. 177-180, 2014.

[5] A. H. Abdelrahman, A. Z. Elsherbeni, and F. Yang, "High-gain and broadband transmitarray antenna using triple-layer spiral dipole elements," IEEE Antennas Wireless Propag. Lett., vol. 13, pp. 12881291, Jul. 2014.

[6] Y. Chen, L. Chen, J.-F. Yu, and X.-W. Shi, "A C-band flat lens antenna with double-ring slot elements," IEEE Antennas Wireless Propag. Lett., vol. 12, pp. 341-344, 2013.

[7] M. Euler and V. F. Fusco, "Frequency selective surface using nested split ring slot elements as a lens with mechanically reconfigurable beam steering capability," IEEE Trans. Antennas Propag., vol. 58, no. 10, pp. 3417-3421, Oct. 2010.

[8] R. Milne, "Dipole array lens antenna," IEEE Trans. Antennas Propag., vol. AP-30, no. 4, pp. 704-712, Jul. 1982.

[9] J. R. Reis et al., "FSS-inspired transmitarray for two-dimensional antenna beam steering," IEEE Trans. Antennas Propag., vol. 64, no. 6, pp. 2197-2206, Jun. 2016.

[10] C. G. M. Ryan, M. Reza, J. Shaker, J. R. Bray, Y. M. M. Antar, and A. Ittipiboon, "A wideband transmitarray using dual-resonant double square

rings," IEEE Trans. Antenna Propag., vol. 58, no. 5, pp. 1486-1493, May 2010

[11] P.-Y. Feng, S.-W. Qu, S. Yang, L. Shen, and J. Zhao, "Ku-band transmitarrays with improved feed mechanism," IEEE Trans. Antennas Propag., vol. 66, no. 6, pp. 2883-2891, Feb. 2018.

[12] P.-Y. Feng, Qu S W. 60-GHz 2D Scan Phased Transmitarray with High Gain and Low Profile[C]//2019 13th European Conference on Antennas and Propagation (EuCAP). IEEE, 2019: 1-4.

[13] P. Feng and S. Qu, "A novel three-layer linearly polarized wideband transmitarray antenna," 2018 IEEE International Symposium on Antennas and Propagation \& USNC/URSI National Radio Science Meeting, Boston, MA, 2018, pp. 1767-1768.

[14] S. Datthanasombat, "Analysis and design of high-gain space-fed passive microstrip array antennas," Doctoral dissertation, Univ. of Southern California, Los Angeles, CA, USA, 2003.

[15] S. Datthanasombat, A. Prata, Jr., L. R. Amaro, J. A. Harrell, S. Spitz, and J. Perret, "Layered lens antenna," in Proc. IEEE Antennas Propag. Soc. Int. Symp., Boston, MA, USA, 2001, pp. 777-780.

[16] D. M. Pozar, Microwave Engineering, 3rd ed. New York, NY, USA: Wiley, 2005.

[17] C. A. Balanis, Adv. Eng. Electromagn., 2nd ed. New York, NY, USA: Wiley, 2012. 